\title{
Avaliação Aerodinâmica do Retrovisor de um Veículo Hatchback usando o Método Adjoint de Otimização
}

\author{
Felipe Magazoni ${ }^{1.2}$, Filipe Fabian Buscariolo ${ }^{1.3}$, Flavio Maruyama ${ }^{1}$, Julio Cesar Lelis Alves ${ }^{1}$ e \\ Leonardo D. Volpe ${ }^{1}$ \\ ${ }^{1} \mathrm{GM}$ Brasil \\ ${ }^{2} \mathrm{NETeF}-\mathrm{USP}$ \\ ${ }^{3} \mathrm{NDF}-U S P$
} E-mails: felipe.magazoni@gm.com, filipe.buscariolo@gm.com, flavio.maruyama@gm.com,
julio.alves@gm.com, leonardo.volpe@gm.com

\section{RESUMO}

Hoje em dia, um dos fatores mais importantes no desenvolvimento de veículos é o aspecto aerodinâmico. Várias iniciativas ao redor do mundo regulam emissões de poluentes e eficiências de veículos tais como a EURO para o mercado europeu e o Projeto INOVAR que será implementado no Brasil em 2017. Assim, este estudo tem como objetivo realizar uma otimização a fim de reduzir a força de arrasto em um veículo Hatchback. O principal objetivo deste trabalho é demonstrar o potencial das técnicas de otimização para melhorar o perfil aerodinâmico do retrovisor do lado do motorista de um veículo Hatchback. O método de otimização usado neste trabalho é o Adjoint, que realiza uma análise de sensibilidade da superfície do veículo e uma reestruturação das malhas superficiais e volumétricas. O estudo é feito usando simulações CFD para reduzir o arrasto aerodinâmico de um veículo Hatchback atual de produção, que foi previamente validado e correlacionado em um teste de túnel de vento. Primeiramente, o método de otimização é utilizado para melhorar a superfície do retrovisor a fim de reduzir a força de arrasto. Os resultados mostram que o coeficiente de arrasto do veículo é reduzido em 0.008. Este trabalho demonstra o potencial das técnicas de otimização, que podem ser aplicadas em estágios iniciais do projeto e em diversas superfícies do veículo.

\section{INTRODUÇÃO}

Os projetos de carros atuais buscam um melhor rendimento no quesito de consumo de combustível e a aerodinâmica desempenha um papel muito importante para atingir esse objetivo. Cada componente de veículo é estudado, visando atender esse requerimento. Para carros de performance, a economia de combustível é desejável, mas velocidade e estabilidade são mais importantes que consumo de combustível e podem ser alcançados com a combinação de potência de motor e desenvolvimento aerodinâmico.

De acordo com HUCHO (1998) [10], 45\% da força de arrasto aerodinâmica é gerada pela parte superior do veículo. $\mathrm{O}$ assoalho e a caixa de rodas são responsáveis pela maior parte da força de arrasto, aproximadamente 55\%. BATCHELDER (2009) [1] apresentou que os retrovisores representam cerca de $3.7 \%$ do coeficiente de arrasto total do veículo. A Figura 1 descreve o consumo de combustível de um veículo comercial. Observe que a parte aerodinâmica representa 
$20 \%$ do consumo de combustível total do veículo e os outros parâmetros são representados pelo tipo de trajeto, condições meteorológicas, engrenamento, motorista e manutenção do veículo.

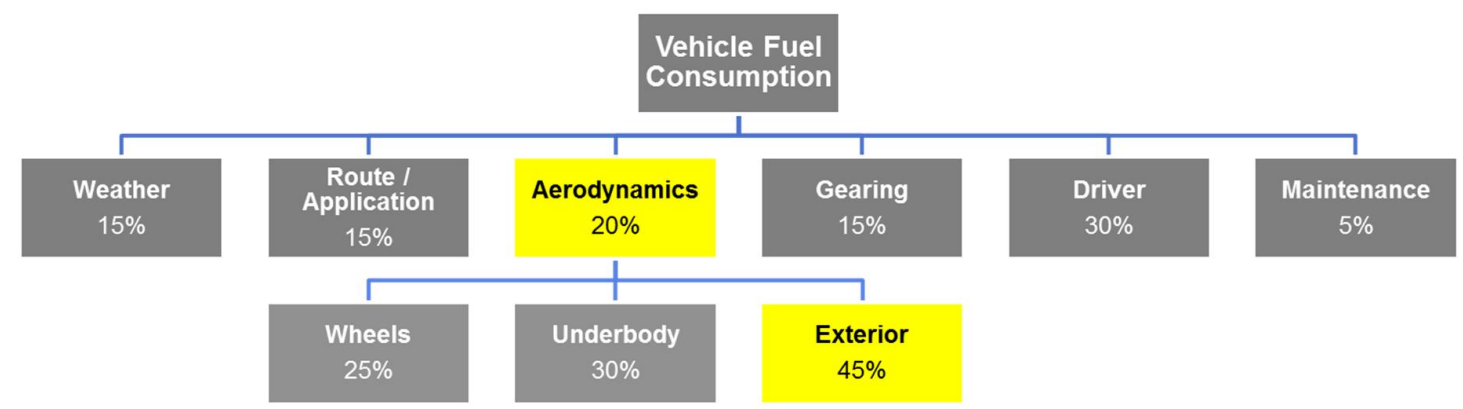

Figura 1. Consumo de combustível de um veículo comercial

Várias iniciativas ao redor do mundo regulam emissões de poluentes e eficiências de veículos tais como a EURO para o mercado europeu e o Projeto INOVAR que será implementado no Brasil em 2017. A EURO possui metas de emissão para todos os tipos de veículos, entretanto neste estudo, somente carros de passeio será considerado. De acordo com os estudos realizados por EUROPEAN ENVIRONMENT AGENCY (2014) [6], para carros de passeio, a meta de emissão de $\mathrm{CO}_{2}$ é de $130 \mathrm{~g} \mathrm{CO}_{2} / \mathrm{km}$ em 2015. Em 2021, a meta de emissão de poluentes será de $95 \mathrm{~g} \mathrm{CO}_{2} / \mathrm{km}$.

O projeto INOVAR é um programa em que os fabricantes de automóveis precisam melhorar a eficiência média do veículo em aproximadamente 12\% até outubro de 2017 em relação aos níveis de 2012. Esta meta foi baseada nas metas de 2015 do mercado europeu de $130 \mathrm{gCO}_{2} / \mathrm{km}$, e foram adaptadas às condições do Brasil baseadas nas diferenças de veículo, combustível e estradas. Para qualificar para uma redução no IPI de $1 \%$ e $2 \%$, os fabricantes de automóveis precisam alcançar uma eficiência média do veículo em outubro de 2016 , que resultarão em uma melhoria média na eficiência de novos veículos de $16 \%$ e $19 \%$, respectivamente.

Para alcançar estas metas, os fabricantes de automóveis investem em CFD - Computational Fluid Dynamics, e em testes de túneis de vento com o objetivo de otimizar superfícies exteriores e analisar o comportamento aerodinâmico. Devido ao alto nível de correlação e à possibilidade de trabalhar em estágios inicias do projeto veicular, a simulação tem sido largamente utilizada nas indústrias automobilísticas.

Alguns trabalhos foram desenvolvidos a fim de analisar a influência da abertura da roda no coeficiente de arrasto para veículos sedan de alta performance (Buscariolo e Karbon, 2011 [2]). $\mathrm{O}$ estudo foi realizado utilizando as ferramentas de CFD com o objetivo de análise do comportamento aerodinâmico das rodas.

Um novo procedimento utilizado em otimização, chamado de Adjoint, vem sendo desenvolvido e utilizado com mais frequência nas simulações computacionais. O método Adjoint calcula derivadas de parâmetros de engenharia, tais como força de arrasto, em função de todos os parâmetros de entrada e fornece uma orientação mais direta para modificações na otimização de superfícies.

Um grande número de trabalhos de otimização vem sendo desenvolvido utilizando a metodologia do Adjoint. Uma revisão bibliográfica dos desenvolvimentos recentes na 
otimização é apresentada por GILES e PIERCE (2000) [7]. Detalhes sobre a construção das equações do método de otimização, suas condições de contorno e o significado físico da solução do Adjoint são descritos no trabalho de GILES e PIERCE (2000) [7].

ELLIOTT (1998) [5] e NIELSEN (1998) [12] derivaram e implementaram a formulação exata do Adjoint das equações de Euler para volumes finitos com malhas não estruturadas. A abordagem do método Adjoint foi desenvolvida baseada na solução das equações de NavierStokes. Alguns exercícios de otimização foram apresentados, e demonstraram a efetividade e a praticidade do sistema de otimização em encontrar a melhor geometria para um determinado escoamento. Por exemplo, o método Adjoint foi utilizado para reduzir a força de sustentação de perfis de aerofólios NACA. A metodologia Adjoint também foi utilizada na redução da força de arrasto para uma força de sustentação constante de uma asa Onera-M6 (Carpentieri, 2009) [3]. Para tal resultado, foram necessárias 28 iterações a fim de reduzir a força de arrasto em $23 \%$.

HAN et al. (2011) [8] e HAN et al. (2012) [9] apresentaram a equação discretizada do método Adjoint para o programa ANSYS-Fluent e resolveram um problema para fornecer uma análise de sensibilidade de superfícies em função da força de arrasto para um veículo com geometria simplificada. Os autores compararam o método Adjoint com o método de gradiente de pressão e concluíram que o método Adjoint é mais explícito e mais consistente.

ZAYA (2013) [13] utilizou o método Adjoint desenvolvido no ANSYS-Fluente para otimizar uma superfície de um veículo simplificado. Concluiu-se que o método Adjoint da ANSYS não está totalmente pronto para otimizações aerodinâmicas, por causa de seu contínuo desenvolvimento. Entretanto, hoje em dia existem diversas metodologias que melhoraram o método Adjoint.

Como visto anteriormente, o método Adjoint vem sendo utilizado em diversas aplicações industriais a fim de otimizar superfícies e reduzir a força de arrasto e outros parâmetros. $\mathrm{O}$ principal objetivo deste estudo é usar a técnica de otimização para fornecer uma superfície otimizada do retrovisor do lado do motorista de um veículo Hatchback, que possui uma grande contribuição no coeficiente total de arrasto do carro, uma vez que o retrovisor está diretamente exposto ao escoamento de ar.

\section{FORMULAÇÃO MATEMÁTICA}

É necessário calcular as variáveis associadas ao escoamento baseada nas leis de conservação de massa, de momento e de energia, que é expressa através da expressão abaixo,

$$
\frac{\partial \rho \phi}{\partial t}+\operatorname{div}\left(\rho \phi u_{r}\right)=\operatorname{div}(\Gamma \operatorname{grad} \phi)+S_{\phi}
$$

onde $\rho$ é a massa específica do fluido, $t$ é o tempo, $u_{r}$ é a velocidade relativa entre o fluido e o sistema de coordenada, $\phi$ é uma variável escalar $(u, v, w, k, \varepsilon, E), \Gamma$ é o coeficiente de difusividade e $S_{\phi}$ é o termo fonte.

Substituem-se as variáveis escalares na Equação 1 e rearranjam-se a expressão, usando as equações de Navier-Stokes, que descrevem o movimento do fluido e suas propriedades, e 
considerando o escoamento incompressível, fluido Newtoniano isotérmico (massa específica e viscosidade são constantes), tem-se,

$$
\begin{gathered}
\nabla \cdot V=0 \\
\rho \frac{D V}{D t}=-\nabla P+\rho g+\mu \nabla^{2} V
\end{gathered}
$$

onde $P$ é a pressão, $\mu$ é a viscosidade dinâmica, $V$ é o campo de velocidade e $g$ é a aceleração da gravidade.

Como as equações de Navier-Stokes compõem um sistema aberto para ser computacionalmente resolvido, um modelo de turbulência é necessário para ser incorporado ao sistema. Neste trabalho, o modelo $k$ - $\varepsilon$ é considerado para resolver as equações de Navier-Stokes. O modelo de turbulência $k-\varepsilon$ é composto por duas equações que vem diretamente das formulações diferencias de transporte, em que $k$ representa a energia cinética turbulenta e $\varepsilon$ representa a taxa de dissipação turbulenta.

$$
\begin{gathered}
\frac{\partial \rho k}{\partial t}+\operatorname{div}\left(\rho k u_{r}\right)=\operatorname{div}\left(\frac{\mu_{t}}{\sigma_{k}} \operatorname{grad} k\right)+2 \mu_{t} E_{i j} E_{i j}-\rho \varepsilon \\
\frac{\partial \rho \varepsilon}{\partial t}+\operatorname{div}\left(\rho \varepsilon u_{r}\right)=\operatorname{div}\left(\frac{\mu_{t}}{\sigma_{\varepsilon}} \operatorname{grad} \varepsilon\right)+C_{1 z} \frac{\varepsilon}{k} 2 \mu_{t} E_{i j} E_{i j}-C_{2 z} \rho \frac{\varepsilon^{2}}{k}
\end{gathered}
$$

onde $\mu_{t}$ é a viscosidade turbulenta, $C_{1 z}, C_{2 z}, \sigma_{\varepsilon}$ e $\sigma_{k}$ são as constantes do modelo de turbulência.

De acordo com HUCHO (1998) [10], a força trativa Ft do veículo requerida na interface entre os pneus e o asfalto é dado por,

$$
F t=D+R+m \frac{d V}{d t}+m g \sin \alpha
$$

onde $D$ é a força aerodinâmica, $R$ é a resistência do rolamento, $m$ é a massa do veículo, $V$ é a velocidade do veículo e $\alpha$ é a ângulo de inclinação da rodovia.

A força aerodinâmica depende de diversos fatores como o tamanho do veículo, que é representado pela área frontal $A$, o coeficiente de arrasto $c_{D}$ e a velocidade do veículo $V$, e é apresentada pela formulação abaixo,

$$
D=\frac{\rho}{2} V^{2} c_{D} A
$$

A Equação 7 pode derivar diversas outras funções dependentes da velocidade do vento e sua direção. O método de otimização é usado para reduzir o coeficiente de arrasto e consequentemente diminuir a força aerodinâmica e a força trativa. Estas otimizações trazem diversos benefícios ao veículo, como por exemplo a redução do consumo de combustível. 
De acordo com CARPENTIERI (2009) [3], para resolver um problema de otimização, primeiramente, é necessário a solução do escoamento para que as variáveis de interesse de otimização, tais como arrasto ou sustentação, possam sem calculadas.

A fim de desenvolver as formulações do método Adjoint, considera-se a função $J=J\left(U, \alpha_{i}\right)$, que depende das variáveis do escoamento $U$ e dos parâmetros geométricos $\alpha_{i}$, onde $i=1, M$. Na prática a variável $J$ pode ser a força de sustentação, o arrasto ou algum outro parâmetro similar. As variáveis do escoamento são a solução das equações do escoamento em regime permanente $R p(U, \alpha)=0$, onde $R p$ é o vetor residual, que também depende das mesmas variáveis. Diferenciando a função residual em função de $\alpha_{i}$, tem-se,

$$
\frac{d J}{d \alpha_{i}}=\frac{\partial J}{\partial \alpha_{i}}+\frac{\partial J}{\partial U} \frac{\partial U}{\partial \alpha_{i}}
$$

onde $\partial U / \partial \alpha_{i}$ é a sensibilidade do escoamento, que pode ser calculada através da linearização da equação. O gradiente da função residual é,

$$
\frac{\partial R p}{\partial U} \frac{\partial U}{\partial \alpha_{i}}=-\frac{\partial R p}{\partial \alpha_{i}}
$$

A Figura 2 apresenta o diagrama de resolução do processo de otimização utilizando a metodologia do Adjoint (Carpentieri, 2009) [3]. A otimização possui uma certa quantidade de processos iterativos a fim de encontrar a melhor geometria para um determinado problema. As etapas deste processo são,

1. Resolver o problema de mecânica dos fluidos e pós-processar os resultados da maneira usual;

2. Escolher uma variável de interesse de engenharia para realizar a otimização;

3. Configurar e resolver o método de otimização para esta variável de interesse;

4. Pós-processar a solução do método Adjoint para verificar a sensibilidade da geometria e das condições de contorno;

5. Realizar uma reestruturação da malha baseada na análise de sensibilidade da superfície;

6. Resolver novamente o problema de mecânica dos fluidos e verificar os resultados;

7. Caso necessário, é possível recomeçar o processo de otimização.

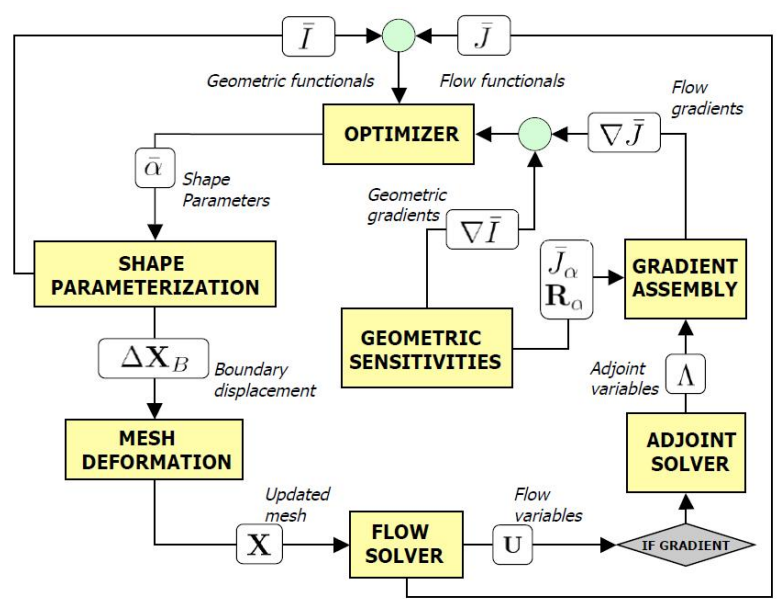

Figura 2. Diagrama de resolução do processo de otimização (Carpentieri, 2009) [3] 


\section{DETALHES DO TÚNEL DE VENTO}

Para realizar o estudo de simulação CFD, um túnel de vento similar ao da General Motors (GM), um dos maiores túneis do mundo, foi considerado. O túnel consiste de um sistema de circuito fechado com controle de temperatura utilizado para realizar os estudos aerodinâmicos tais como estabilidade direcional e conforto térmico em diversos tipos de veículos. A seção de teste possui piso estático com um sistema de sucção da camada limite no início da seção.

De acordo com KELLY et al. (1982) [11], o túnel da GM apresenta uma área de seção de 56.16 $\mathrm{m}^{2}$ e um comprimento de aproximadamente $21.3 \mathrm{~m}$. É construído de metal revestido por uma camada de concreto de $600 \mathrm{~mm}$ com o objetivo de prevenir a propagação de som. O escoamento de ar é realizado por um poderoso ventilador de $13 \mathrm{~m}$ de diâmetro e uma potência de aproximadamente $3000 \mathrm{~kW}$, capaz de alcançar velocidades de até $250 \mathrm{~km} / \mathrm{h}$ por 15 minutos. Um esquema do túnel de vento da GM é apresentado na Figura 3.

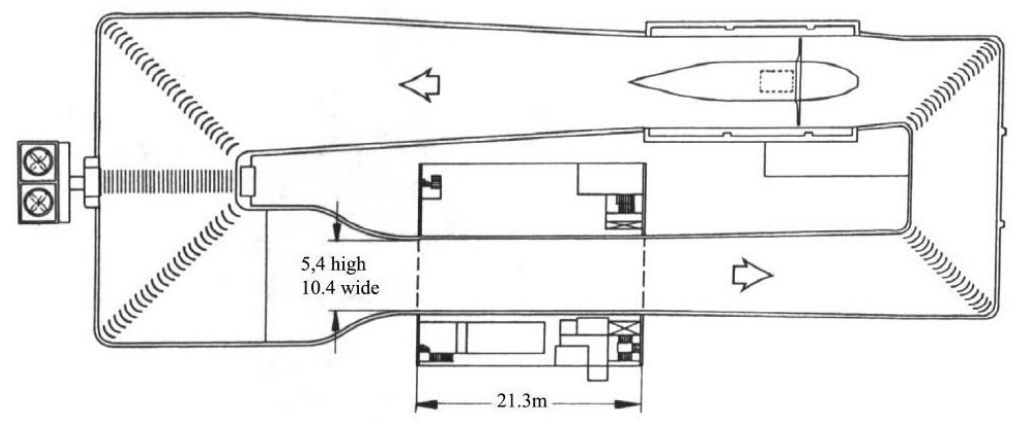

Figura 3. Túnel de vendo da General Motors (Hucho, 1998) [10]

O túnel de vento da GM possui piso estático combinado com um sistema de sucção de camada limite para reduzir seu efeito sobre o coeficiente de arrasto do carro. Muitos estudos vêm sendo realizados para entender o comportamento do sistema de sucção de camada limite no túnel de vento e como evitar sua interferência nas medições aerodinâmicas. Neste modelo, não existe rotação das rodas, que podem interferir no escoamento ao redor do veículo.

O método que melhor aproxima às condições reais de um veículo é aplicando esteira rolante no piso da seção de teste do túnel de vento. A ideia é reproduzir a condição de uma estrada, onde o veículo está se locomovendo contra o ar. Para que tal condição ocorra no túnel de vento, a esteira deve se movimentar com a mesma velocidade do ar. As rodas do veículo devem também se movimentar e estar em contato com a esteira. Neste presente trabalho, esta aproximação será utilizada no desenvolvimento das simulações.

\section{METODOLOGIA}

Neste trabalho, o processo de otimização do retrovisor lateral do lado do motorista é realizado usando o método Adjoint do FLUENT®. O túnel de vento deste trabalho possui dimensões similares ao túnel da General Motors (GM), localizado em Warren, Estados Unidos. Os parâmetros de otimização são estudados no túnel de vento, onde o ar passar ao longo do veículo, simulando seu movimento. 
Assim como no trabalho de BUSCARIOLO e KARBON (2011) [2], os mesmos parâmetros são utilizados para a simulação computacional, e são expressos na tabela abaixo,

Tabela 1. Parâmetros da simulação computacional

\begin{tabular}{|l|c|}
\hline Túnel de vento & General Motors, Warren-Michigan \\
\hline Velocidade do ar & $110 \mathrm{~km} / \mathrm{h}(30.56 \mathrm{~m} / \mathrm{s})$ \\
\hline Pressão de saída & Pressão atmosférica \\
\hline Intensidade da turbulência & $0.60 \%$ \\
\hline Sistema de sucção da camada limite & No início da seção de teste \\
\hline Dimensões da seção do teste & $(5.4 \times 10.4 \times 23.0) \mathrm{m}$ \\
\hline Modelo de turbulência & $k-\varepsilon$ \\
\hline
\end{tabular}

As simulações foram realizadas utilizando o programa FLUENT®. Hypermesh® é usado na construção da malha superficial do veículo e do túnel de vento; T-Grid® é usado para gerar a malha volumétrica. O veículo de otimização é um Hatchback, representado por um modelo virtual e considerando todas as partes do motor do veículo e a parte do sistema de arrefecimento. O modelo do veículo pode ser visualizado na Figura 4.

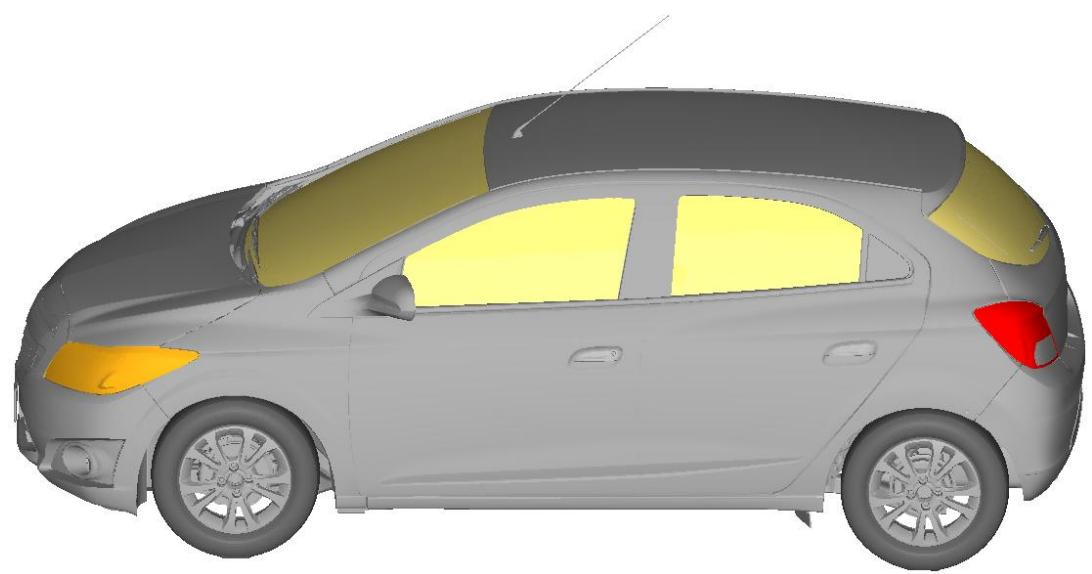

Figura 4. Representação do veículo estudado neste trabalho

O total de elementos volumétricos da malha, considerando um tamanho de malha superficial média de $10 \mathrm{~mm}$, é de aproximadamente 60 milhões, levando em conta o túnel de vento. $\mathrm{O}$ tempo total de processamento é de aproximadamente 30 horas utilizando 288 CPUs.

\section{RESULTADOS}

A técnica de otimização é feita utilizando o ANSYS-Fluent com foco na melhoria geométrica do retrovisor lateral do lado do motorista. O principal objetivo da otimização é reduzir a força axial no veículo, e consequentemente diminuir o coeficiente de arrasto. Primeiramente, uma simulação com os parâmetros apresentados na Tabela 1 é realizada. A força total e o coeficiente de arrasto encontrados são 508.97 N e 0.414, respectivamente. É importante ressaltar que este primeiro cálculo precisa convergir muito bem para que os resultados do processo de otimização não sejam prejudicados.

Uma análise de sensibilidade é realizada utilizando o método Adjoint com o objetivo de extrair os parâmetros de sensibilidade da força axial em relação à geometria. A Figura 5 indica a localização do retrovisor lateral onde pequenas alterações da superfície geométrica provocam 
grandes mudanças na força axial. Em algumas regiões, a magnitude da sensibilidade é menor, indicando que alterações geométricas ocasionam pequenas mudanças na força axial. A Figura 5 apresenta o comportamento da magnitude da sensibilidade no retrovisor lateral do lado do motorista. As regiões em vermelho representam a localização onde pequenas modificações produzirão grandes alterações na força axial e é esta região onde o processo de reestruturação de malha irá trabalhar. Como observado, a borda externa do retrovisor lateral é mais susceptível na redução da força axial e consequentemente do coeficiente de arrasto.
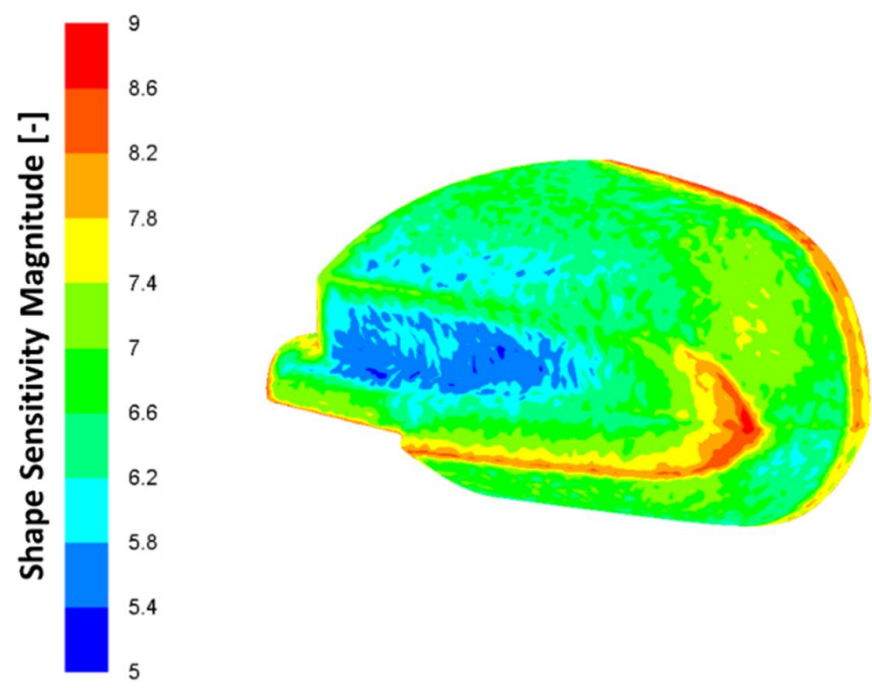

Figura 5. Magnitude da sensibilidade na superfície do retrovisor lateral do lado do motorista

Assim, é possível modificar a geometria baseado nos resultados de sensibilidade utilizando os métodos de reestruturação de malha do FLUENT. A malha volumétrica da região a ser reestruturada é dividida em 25 pontos em cada sistema de coordenada. A escala de deformação da malha é escolhida movendo os pontos de controle proporcionalmente aos resultados de sensibilidade. O processo fornece um reposicionamento suave não somente da malha superficial, mas também da malha volumétrica, respeitando alguns critérios de geração de malha. A superfície otimizada pode ser vista na Figura 6.

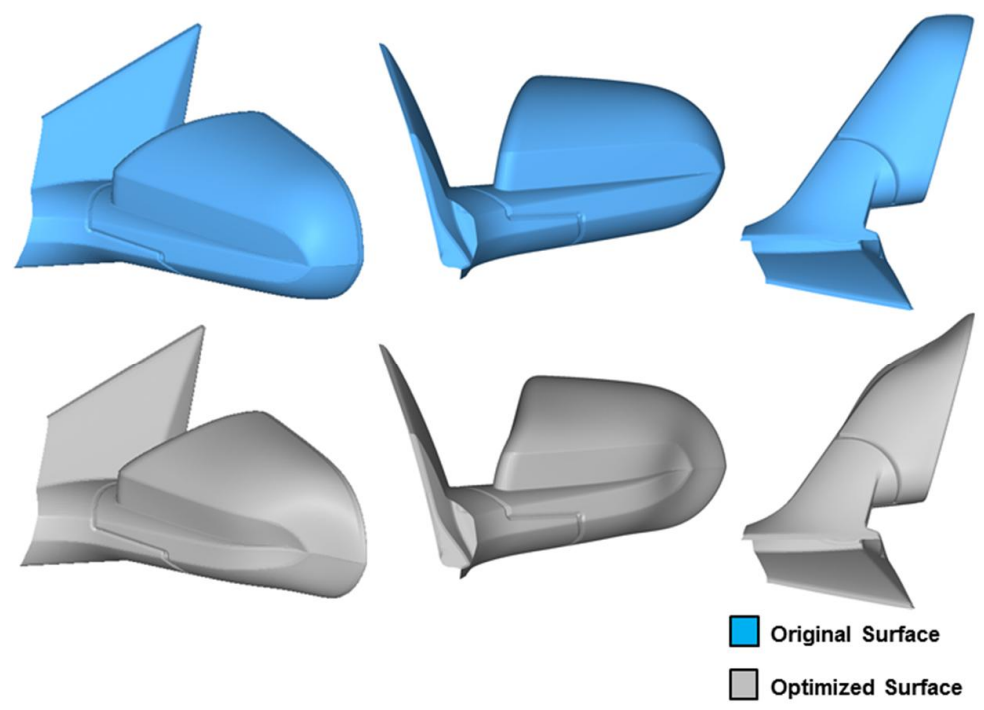

Figura 6. Superfícies original e otimizada do retrovisor lateral do lado do motorista 
A Tabela 2 apresenta os resultados da força total e do coeficiente de arrasto das superfícies original e otimizada do retrovisor lateral do lado do motorista. A força total da superfície original é de $508.97 \mathrm{~N}$, e a técnica de otimização fez com que a força reduzisse $9.98 \mathrm{~N}$, que representa 0.008 do coeficiente de arrasto do veículo.

Tabela 2. Resultados das superfícies original e otimizada

\begin{tabular}{|l|c|c|c|}
\cline { 2 - 4 } \multicolumn{1}{c|}{} & $\begin{array}{c}\text { Superfície } \\
\text { Original }\end{array}$ & $\begin{array}{c}\text { Superfícies } \\
\text { Otimizada }\end{array}$ & Diferença \\
\hline Coefica Total [N] & 508.97 & 498.99 & 9.98 \\
\hline
\end{tabular}

As Figuras 7 e 8 apresentam o comportamento da energia cinética turbulenta ao longo da esteira do veículo. A otimização do retrovisor lateral gera uma modificação do escoamento ao redor da traseira do carro e do retrovisor, que faz com que o coeficiente de arrasto diminua. Como observado, existe uma turbulência maior na traseira do carro da superfície original do veículo, que define uma zona de separação de escoamento. A superfície otimizada reduz esta turbulência e diminui a força total do veículo.
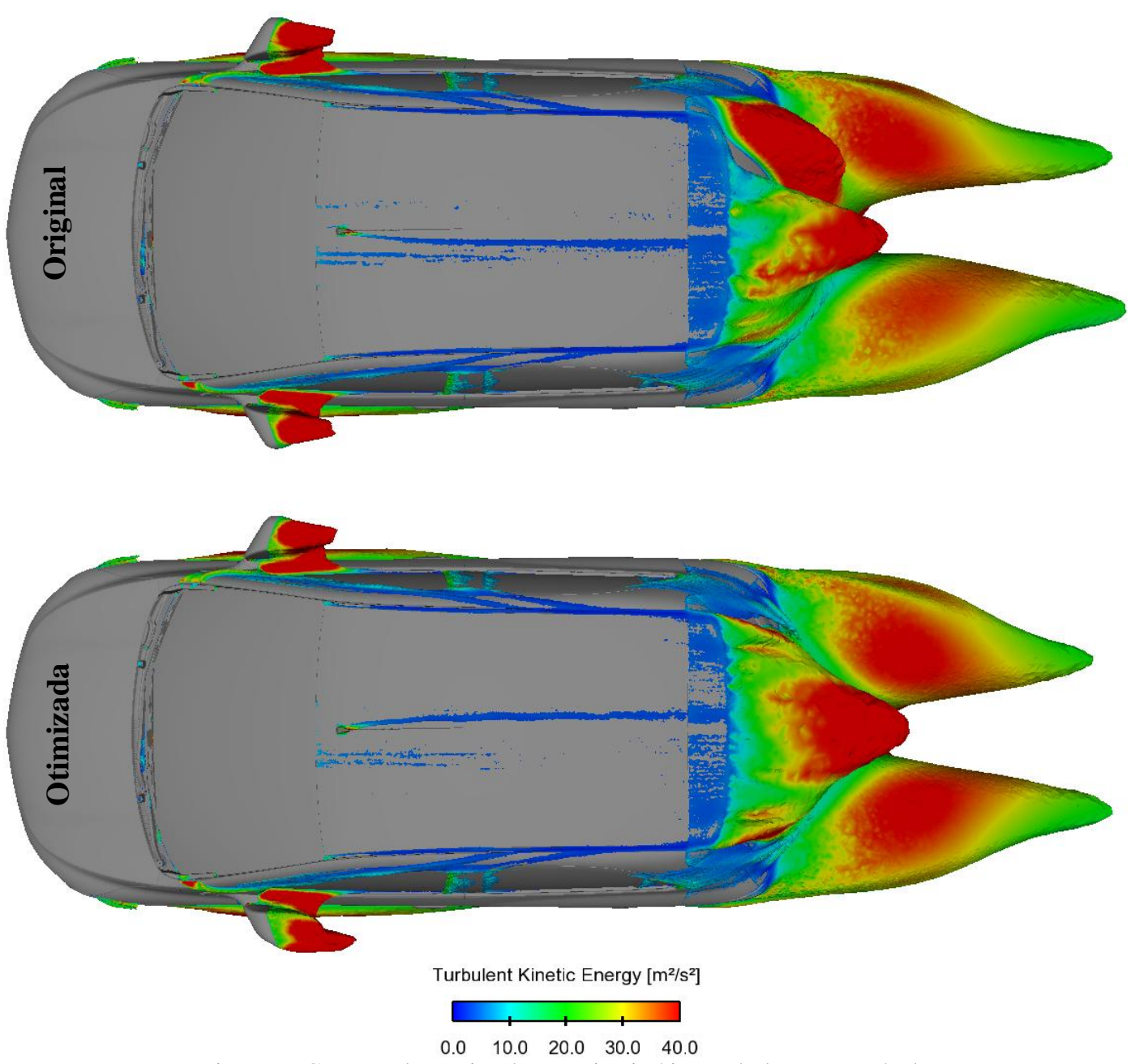

Figura 7. Contour da esteira de energia cinética turbulenta no veículo 

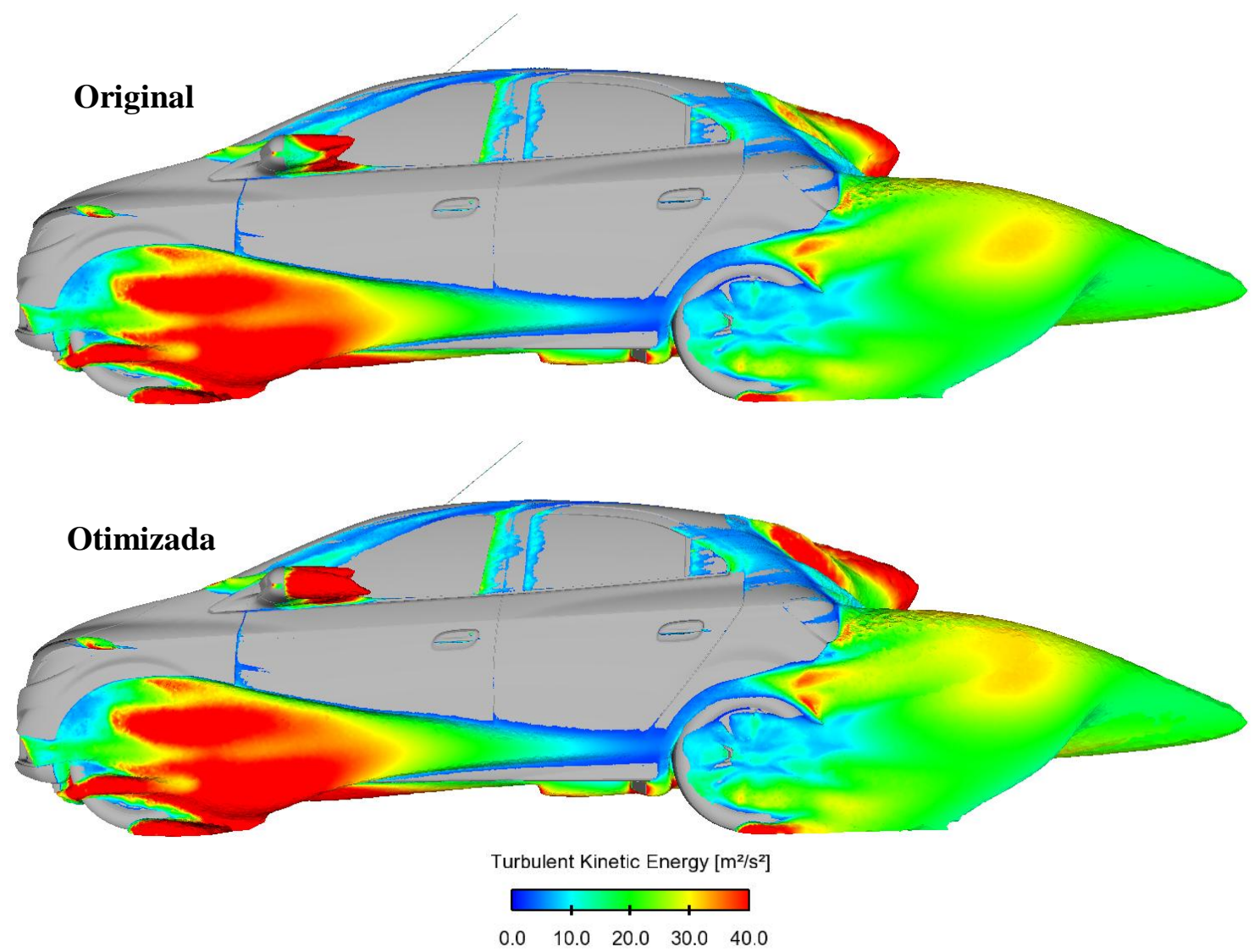

Figura 8. Contour de energia cinética turbulenta no veículo

A Figura 9 apresenta o comportamento da pressão estática na superfície próxima ao retrovisor lateral do lado do motorista. As regiões nas quais a pressão estática é negativa são indicadas pela cor azul e verde claro. Como visto na figura, existe uma região da superfície original com altos valores de pressão estática. É gerado um ponto de estagnação na frente e uma separação na traseira do retrovisor, que fazem com que o coeficiente de arrasto aumente. 


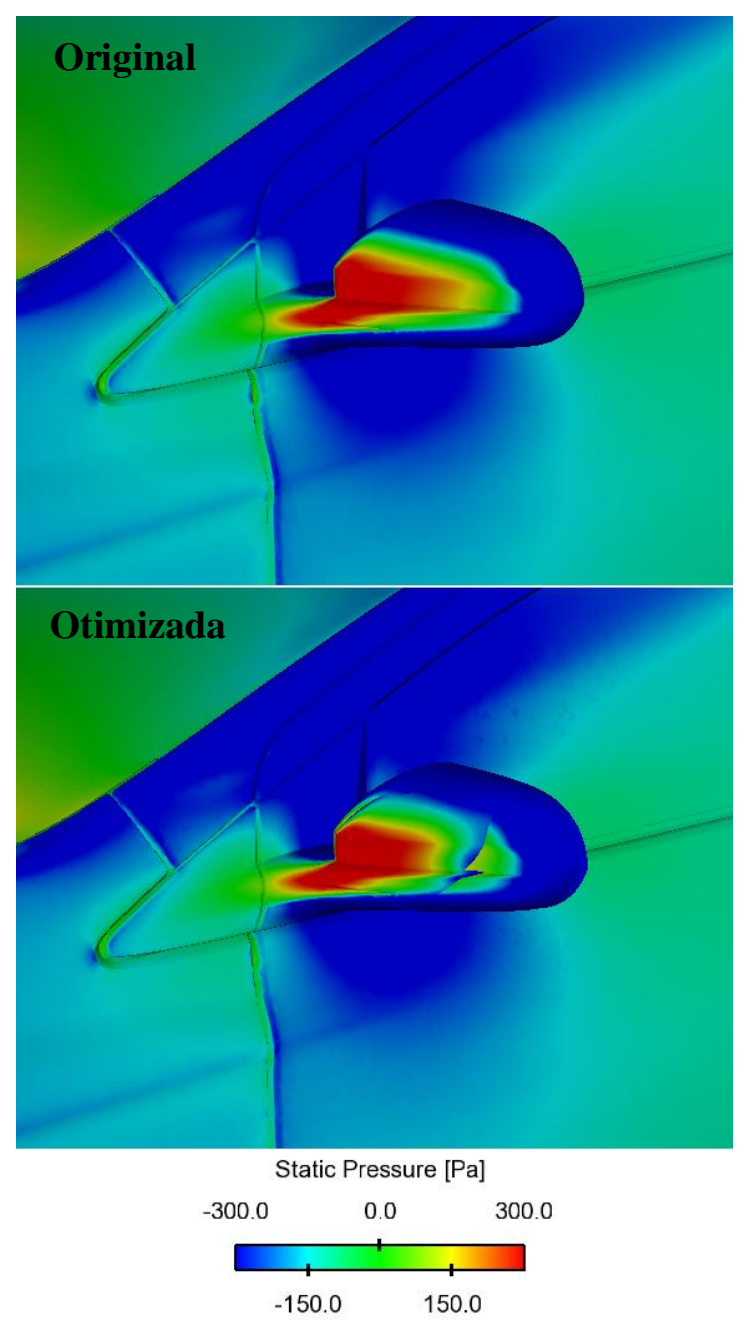

Figura 9. Detalhes da pressão estática no retrovisor lateral do lado do motorista

\section{CONCLUSÃO}

Este trabalho realiza um processo de otimização com o objetivo de reduzir a força de arrasto em veículos Hatchback. O principal objetivo do trabalho é demonstrar o potencial das técnicas de otimização na realização de melhorias aerodinâmicas do retrovisor lateral do lado do motorista. A metodologia de otimização utilizada neste presente estudo é o método Adjoint.

A análise de sensibilidade é realizada no retrovisor lateral e os resultados demonstram o potencial de modificações que podem ser feitas no veículo. Esta análise indica as localizações da superfície do retrovisor lateral onde pequenas alterações provocam grandes mudanças na força axial. A borda do retrovisor lateral é o local mais susceptível para a redução da força axial e consequentemente do coeficiente de arrasto.

A superfície otimizada é gerada utilizando os métodos de reestruturação de malha e dos resultados da análise de sensibilidade. A força total reduziu em $9.98 \mathrm{~N}$, que representa 0.008 do coeficiente de arrasto. 
Este trabalho demonstra o potencial das técnicas de otimização, que podem ser aplicadas em estágios inicias de projeto e podem ser aplicadas em diversas superfícies do veículo. A otimização Adjoint vem recebendo grandes atenções como um excelente método de análise de sensibilidade para questões aerodinâmicas e também para redução de perdas de cargas (Han et al., 2011 [8] e Han et al., 2012 [9]).

\section{NOMENCLATURA}

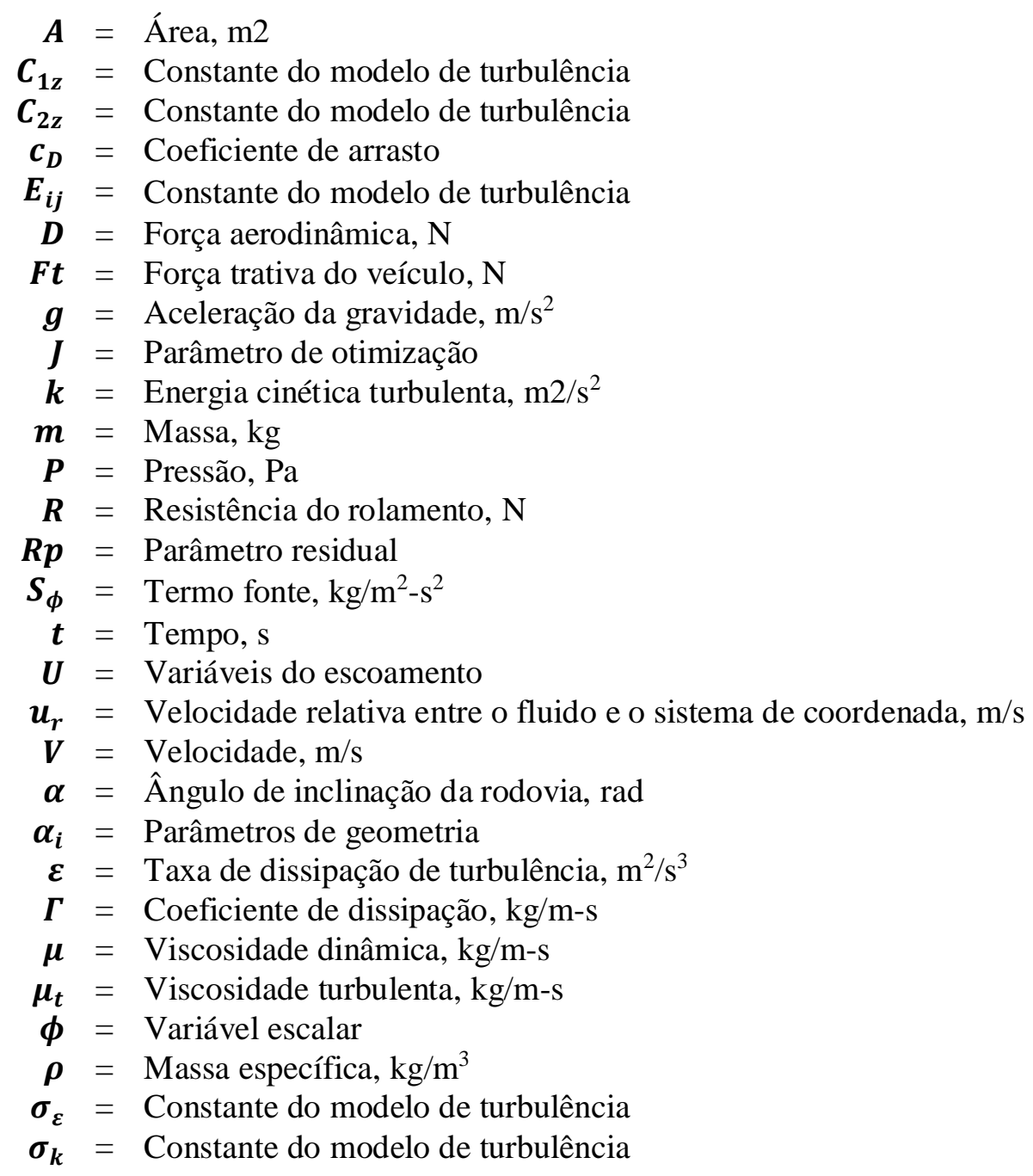

\section{REFERÊNCIAS}

[1] BATCHELDER, J. H., A CFD Investigation of Potential Aerodynamic Enhancements to a Microcar Class Vehicle. Master, Faculty of Rensselaer Polytechnic Institute, 2009. 
[2] BUSCARIOLO, F. F.; KARBON, K. J., Comparative CFD Analysis Between Rotating and Static Cases of Different Wheels Opening Designs over a Performance Sedan, SAE Technical Paper, 2011.

[3] CARPENTIERI, G., An Adjoint-Based Shape-Optimization Method for Aerodynamic Design, Ph.D. Thesis, Technische Universiteit Delft, 2009.

[4] CARPENTIERI, G.; KOREN, B.; VAN TOOREN, M. J. L., Adjoint-based Aerodynamic Shape Optimization on Unstructured Meshes, Journal of Computational Physics, 224, 267-287, 2007.

[5] ELLIOTT J., Aerodynamic optimization based on the Euler and Navier-Stokes equations using unstructured grids. Ph.D. Thesis, MIT, Department of Aeronautics and Astronomy, 1998.

[6] European Environment Agency, Monitoring $\mathrm{CO} 2$ emissions from passenger cars and vans in 2013, EEA Technical report No 19/2014, 2014.

[7] GILES, M. B.; PIERCE N. A., An Introduction to the Adjoint Approach to Design, Flow, Turbulence and Combustion, 65, 393-415, 2000.

[8] HAN, T.; HILL, C.; JINDAL, S., Adjoint Method for Aerodynamic Shape Improvement in Comparison with Surface Pressure Gradient Method, SAE Technical Paper, 2011.

[9] HAN, T.; KAUSHIK, S.; GIN, R.; BOT, E., Adjoint Method for Aerodynamic Shape Improvement, SAE Technical Paper, 2012.

[10] HUCHO, W. H., Aerodynamics of Road Vehicles, From Fluid Mechanics of Vehicle Engineering, 4th Edition, SAE International, 1998.

[11] KELLY, K. B.; PROVENCHER, L. G.; SCHENKEL, F. K., The General Motors Engineering Staff Aerodynamics Laboratory - A full Scale Automotive Wind Tunnel, SAE Paper, No 820371, Society of Automotive Engineers, 1982.

[12] NIELSEN, E. J.; ANDERSON, W. K., Aerodynamic Design Optimization on Unstructured Meshes Using the Navier-Stokes Equations, AIAA Paper 98-4809, 1998.

[13] ZAYA, J., Aerodynamic Optimization of Ground Vehicles with the Use of Fluent's Adjoint Solver, Master's Thesis, Chalmers University of Technology, 2013. 